\title{
Ovarian morphology is associated with insulin resistance in women with polycystic ovary syndrome: a cross sectional study
}

\author{
Sara Pittenger Reid ${ }^{1}$, Chia-Ning Kao ${ }^{1}$, Lauri Pasch ${ }^{1}$, Kanade Shinkai ${ }^{2}$, Marcelle I. Cedars ${ }^{1}$
} and Heather G. Huddleston ${ }^{1 *}$

\begin{abstract}
Background: Polycystic ovary syndrome (PCOS) is a very common disorder well known to be associated with insulin resistance and metabolic disease. Insulin resistance is likely involved in the promotion of the PCOS reproductive phenotype and may mediate some of the ovarian morphology seen in the disorder. The phenotype of each individual woman with PCOS can vary widely as can her metabolic risk.

Methods: This is a cross-sectional study of patients seen in a multidisciplinary PCOS clinic at the University of California at San Francisco between 2006 and 2014.

All participants underwent systematic evaluation with anthropometric measurements, comprehensive skin exam, transvaginal ultrasound and laboratory studies at the time of their initial visit to the clinic. Serum samples were stored and androgen studies were carried out on all stored samples at the University of Virginia. Logistic regression was employed to evaluate the association between ovarian volume or follicle number and metabolic parameters (fasting insulin, HOMA-IR, fasting glucose, $2 \mathrm{~h}$ glucose, waist circumference) and hyperandrogenism (free testosterone, total testosterone, DHEAS, acanthosis nigricans), controlling for age.

Results: Three-hundred thirteen patients seen during the study period met Rotterdam criteria for PCOS and had sufficient measurements for inclusion in our analysis. The odds ratio of elevated HOMA-IR for patients with a maximum ovarian volume $>10 \mathrm{cc}$ was 1.9 compared to those with a maximum ovarian volume of $\leq 10 \mathrm{cc}(95 \% \mathrm{Cl} 1.0-3.4)$. The odds ratio of abnormal fasting insulin for patients with higher ovarian volume was 1.8 (95\% Cl 1.0-3.4) compared with those with lower ovarian volume. Follicle number was not significantly associated with any metabolic parameters.

Conclusions: Increased ovarian volume is associated with markers of insulin resistance in PCOS. In concordance with prior studies, we did not find follicle number to be predictive of metabolic risk. Ovarian volume may serve as a useful tool to aid clinicians in their risk stratification and counseling of patients with PCOS.
\end{abstract}

Keywords: Polycystic ovary syndrome, Ovarian volume, Insulin resistance

\section{Background}

Polycystic ovary syndrome (PCOS) is a very common and heterogeneous disorder. Depending on the criteria used to diagnose PCOS, incidence estimates range from $4-9 \%$ of reproductive age women using the $1990 \mathrm{Na}$ tional Institutes of Health criteria [1-3] and as high as $12-21 \%$ using the 2003 Rotterdam criteria [4-7]. Among

\footnotetext{
* Correspondence: huddlestonh@obgyn.ucsf.edu

${ }^{1}$ Center for Reproductive Health, University of California at San Francisco,

2356 Sutter Street, San Francisco 94115, CA, USA

Full list of author information is available at the end of the article
}

these women, there is a wide range of physical, endocrine and metabolic phenotypes.

It is well known that women with PCOS have an increased risk of insulin resistance, diabetes and metabolic syndrome [8-11]. These metabolic abnormalities are associated with significant long-term morbidity. Insulin resistance, one of the earliest manifestations of metabolic disease, has been demonstrated to contribute significantly to the risk for coronary heart disease (CHD). In a recent National Health and Nutrition Examination Survey (NHANES) study, insulin resistance was shown to 
be a stronger risk factor for CHD than diabetes [12]. It is well accepted that insulin resistance is a major driver of the metabolic phenotype in PCOS and is compounded by obesity $[10,8]$. Insulin resistance likely also plays a role in promoting the PCOS reproductive phenotype as insulin has been shown to increase theca androgen production and may mediate some of the ovarian morphology characteristics commonly seen in PCOS by driving theca cell proliferation [13].

The theca cells in women with PCOS appear to be more responsive to the actions of insulin than those of controls [10]. They secrete more androgens both basally and in response to stimulation by leutinizing hormone $(\mathrm{LH})$ and insulin. Insulin acts as a co-gonadotropin in this case, modulating ovarian steroidogenesis [14].

Screening tests and medical treatments exist to detect and treat those with metabolic disease, but to date, identifying which women with PCOS are at greatest risk has not been straightforward. It would be clinically useful to be able to predict an individual woman's metabolic risk using her clinical phenotype.

There has been much discussion and disagreement surrounding the specific thresholds defining the ovarian criteria for PCOS. The 2003 Rotterdam criteria are currently the most commonly used standard for classifying the morphology of polycystic ovaries [7]. Under Rotterdam, polycystic ovary morphology (PCOM) is defined as a follicle number per ovary of $\geq 12$ and/or an ovarian volume of $>10 \mathrm{cc}$ in at least one ovary. The 2014 Androgen Excess and PCOS Society task force recommended the use of $\geq 25$ follicles and/or a volume of $>10$ cc [15]. Because of the existence of these morphologic criteria, specific ultrasound data regarding follicle number and volume exist for most women diagnosed with PCOS, providing clinicians with physical markers that may potentially be useful in stratifying patient phenotype and assessing future risk.

PCOM can be identified in approximately $30 \%$ of reproductive aged women [16, 17]. In normovulatory women, this finding has not been associated with an increased risk of metabolic disease [18]. In women with PCOS, the implications of PCOM are not entirely clear. In a very small study of 10 women with PCOS, women with PCOM showed slower glucose disappearance than those without PCOM [19]. A subsequent larger study in 240 Italian women failed to show a difference in insulin in women with and without PCOM. This study, however, used a more liberal definition of PCOM of $>9$ follicles or a volume $>7.5 \mathrm{cc}$ [20]. Other studies on this question group follicle number per ovary and ovarian volume into one phenotype for comparisons and use a variety of definitions of PCOM making comparisons difficult [21]. Accordingly, there is no clear data regarding the associations of the individual components of PCOM and metabolic risk.
We designed a study to determine if ovarian volume $(\mathrm{OV})$ and/or follicle number (FN) are independently associated with abnormal metabolic findings in women diagnosed with PCOS. We hypothesized that ovarian morphology but not follicle number would be associated with a significantly higher odds of hyperinsulinemia among women with PCOS.

\section{Methods}

\section{Study population and recruitment}

This is a prospective, cross sectional study in which patients attending the monthly multi-disciplinary PCOS clinic at the University of California at San Francisco (UCSF) were recruited consecutively between 2006 and 2014. Patients are referred to this clinic for evaluation to determine whether or not they meet criteria for a diagnosis of PCOS and for management recommendations. The clinic is specifically targeted toward women who are not currently seeking fertility treatment. Patients were voluntarily consented to participate in the study based on a research protocol approved by the UCSF Committee on Human Research. Participants were included if they met Rotterdam criteria for PCOS, with two out of three of the following features: oligo- or an-ovulation, clinical and/or biochemical hyperandrogenism, and the presence of 12 or more antral follicles in one ovary and/ or a maximum ovarian volume $>10 \mathrm{cc}$ [7]. Participants were excluded if they did not have complete ovarian morphology measurements.

\section{Data collection}

All participants presented for a single visit in which they completed a self-administered questionnaire and underwent systematic evaluation with anthropometric measurements, comprehensive dermatologist's exam, transvaginal ultrasound, and blood work.

\section{Outcome variables}

Outcome variables studied included the following metabolic and androgen measurements. Metabolic: fasting insulin $>19 \mu \mathrm{IU} / \mathrm{mL}$, HOMA-IR greater than 4 [22], fasting glucose $>100 \mathrm{mg} / \mathrm{dL}, 2 \mathrm{~h}$ glucose $>140 \mathrm{mg} / \mathrm{dL}$, waist circumference $>89 \mathrm{~cm}$, and the presence or absence of acanthosis nigricans. Hyperandrogenism: total testosterone $>6.8 \mathrm{pg} / \mathrm{mL}$, free testosterone $>53 \mathrm{ng} / \mathrm{dL}$, abnormal DHEAS with cutoff for abnormal level determined by the references for each individual performing lab, $\mathrm{mFG} \geq 8$, presence of severe acne, and presence of androgenic alopecia.

Participants were asked to abstain from use of oral contraceptive pills and spironolactone for at least 1 month prior to the planned clinic visit. They were also asked to refrain from any type of hair removal, including waxing, plucking or shaving, for 1 month prior to the clinic visit. 
A dermatologist performed a comprehensive skin examination of each patient to assess for cutaneous manifestations of hyperandrogenism and hyperinsulinemia. Hirsutism was reported by a modified FerrimanGallwey (mFG), A score $\geq 8$ was considered abnormal. Patients were evaluated for the presence of other cutaneous findings of PCOS, including acne, androgenic alopecia, and acanthosis nigricans [7, 23, 24]. Acne was categorized as "present" if the dermatologist determined that treatment for acne would be appropriate. Physiologic acne that was rare and would not be treated was considered "absent".

Metabolic testing included a $75 \mathrm{~g}$ 2-h oral glucose tolerance test. The homeostasis model assessment-estimated insulin resistance (HOMA-IR) was calculated by multiplying fasting plasma insulin $\mu \mathrm{IU} / \mathrm{mL}$ (FPI) by fasting plasma glucose $\mathrm{mg} / \mathrm{dL}$ (FPG), then dividing by the constant 405 as described by Matthews [25]. Assay specifics are not reported for the metabolic parameters as they were drawn and analyzed at multiple different labs according to patient insurance coverage.

Serum was collected and stored for all subjects who consented for participation in our biobank. In April 2014 in preparation for our androgen subanalysis, the serum of all consented subjects was analyzed for total testosterone and sex hormone biding globulin (SHBG). Total testosterone and SHBG were measured in singlet at the University of Virginia (UVA) Center for Research and Reproduction Ligand Assay and Analysis Core Laboratory (Charlottesville VA) and free testosterone was calculated using the law of mass action as previously described [26, 27].

\section{Exposure variables}

Our exposure variables were ovarian volume and follicle number. OV $\leq 10 \mathrm{cc}$ was considered normal and $\mathrm{OV}$ $>10$ cc was considered elevated. FN was examined at 2 different cutoffs, $\mathrm{FN}<12$ or $\geq 12$; FN $<25$ or $\geq 25$.

The larger of the two OV and higher FN were utilized for analysis. If one ovary was not measurable due to surgical absence or the presence of a cyst, the measurements of the other ovary were used. If neither ovary could be adequately visualized or if the measurements were absent in the records, the patient was excluded from the analysis.

Each patient's transvaginal ultrasound was performed with one of two attending reproductive endocrinologists (M.I.C., H.G.H.). A Shizmadzu SDU-450XL machine with a variable 4- to $8-\mathrm{mHz}$ vaginal transducer was used to measure the transverse, longitudinal, and anteroposterior diameters of each ovary to calculate OV using the equation for volume of an ellipse. Follicles between 2 and $9 \mathrm{~mm}$ in diameter were counted in each ovary to give the FN.

\section{Covariates}

The covariates measured were age and BMI.

\section{Statistical analysis}

For analyses in which OV and FN were treated as dichotomous variables, Rotterdam criteria or Androgen Excess and PCOS Society criteria for polycystic morphology were used to define the variable as normal or abnormal (OV $\leq 10$ cc or $>10$ cc; FN $<12$ or $\geq 12 ; \mathrm{FN}<25$ or $\geq 25$ ) [7]. Ttests were used to compare means of clinical variables by $\mathrm{OV}$ and FN as dichotomous variables. Logistic regression was used to evaluate the effect of OV and FN as dichotomous and continuous variables on metabolic parameters while controlling for age. The number of observations included for each variable of interest is shown in Tables 1, 2, and 3. All computations were performed using SAS 9.3 for Windows, 32-bit edition.

\section{Assays}

Testosterone was measured by radioimmunoassay (RIA) [Coat-a-Count Kit; Siemens Healthcare Diagnositics; assay sensitivity $0.2-180 \mathrm{nmol} / \mathrm{L}$; intraassay coefficient of variation $(C V)=4.4 \%$; interassay $C V=6.4 \%$. SHBG was measured by Immulite [L2KSH2 Kit; Siemens Healthcare Diagnostics; assay sensitivity 6.1-1500.0 ng/ $\mathrm{dl}$; intraassay $(\mathrm{CV})=2.8 \%$; interassay $\mathrm{CV}=6.5 \%]$. Free testosterone was calculated using the following equation derived from the law of mass action:

$$
[\mathrm{fT}]=\left([\mathrm{T}]-(\mathrm{N} \times[\mathrm{fT}]) /\left\{\mathrm{k}_{\mathrm{sT}}\left(\left[\mathrm{C}_{\mathrm{SHBG}}\right]-[\mathrm{T}]+\mathrm{N}[\mathrm{fT}]\right)\right\}\right.
$$

where $\mathrm{k}_{\mathrm{sT}}=$ affinity constant of SHBG for $\mathrm{T}, \mathrm{N}=\mathrm{k}_{\mathrm{aT}} \mathrm{Ca}$ $+1, \mathrm{k}_{\mathrm{aT}}=$ affinity constant of albumin for $\mathrm{T}$, and $\mathrm{Ca}=$ albumin concentration, assumed to be $4.5 \mathrm{gm} / \mathrm{dL}[26,27]$.

Table 1 Cohort Characteristics

\begin{tabular}{llll}
\hline & Mean (SD) & Age & BMI \\
\hline$\leq 10 c c$ & $6.64(2.0)$ & $28.78(5.9)$ & $28.43(7.2)^{a}$ \\
$N=151$ & $14.11(5.7)$ & $28.20(6.0)$ & $30.33(7.9)^{a}$ \\
$>10 c C$ & & \\
$N=162$ & & $29.00(8.3)$ & $29.73(7.6)$ \\
$<12$ follicles & $8.88(2.4)$ & & \\
$N=34$ & & $28.42(5.7)$ & $29.38(7.7)$ \\
$\geq 12$ follicles & $22.42(9.7)$ & $28.74(6.2)$ & $29.81(7.8)$ \\
$N=279$ & $16.11(4.5)$ & & $28.37(6.9)$ \\
$<25$ follicles & & & \\
$N=86$ & $33.7(9.6)$ & & \\
$\geq 25$ follicles & & & \\
$N=227$ & & & \\
\hline
\end{tabular}

i. For all comparisons, the largest available ovarian volume and single ovary follicle number was used for each subject

${ }^{a}$ Indicates a significant difference in BMI between subjects with a maximum ovarian volume $\leq 10 \mathrm{cc}$ compared to those with a maximum volume $>10 \mathrm{cc}$, $p=0.03$ 
Table 2 Logistic Regression Analysis of Follicle Number and Ovarian Volume as Dichotomous Variables

\begin{tabular}{|c|c|c|c|}
\hline & $\begin{array}{l}\text { Follicle Number } \\
\geq 12 \text { vs }<12\end{array}$ & $\begin{array}{l}\text { Follicle Number } \\
\geq 25 \text { vs }<25\end{array}$ & $\begin{array}{l}\text { Ovarian Volume } \\
>10 \mathrm{cc} \text { vs } \leq 10 \mathrm{cc}\end{array}$ \\
\hline & AOR $(95 \% \mathrm{Cl})$ & AOR $(95 \% \mathrm{Cl})$ & AOR $(95 \% \mathrm{Cl})$ \\
\hline Fasting Insulin $>19 \mu \mathrm{lU} / \mathrm{mL}$ & $2.2(0.7-7.0)$ & $0.9(0.5-1.8)$ & $1.8(1.0-3.4)$ \\
\hline HOMA-IR $>4$ & $1.2(0.4-3.4)$ & $0.8(0.4-1.6)$ & $1.9^{\mathrm{a}}(1.0-3.6)$ \\
\hline Fasting Glucose $>100$ mg/dL & $0.9(0.3-2.9)$ & $0.5(0.2-1.4)$ & $1.8(0.8-4.0)$ \\
\hline $2 \mathrm{~h}$ Glucose $>140 \mathrm{mg} / \mathrm{dL}$ & $0.9(0.3-2.9)$ & $0.4(0.1-1.0)$ & $1.3(0.6-2.7)$ \\
\hline Waist Circ $>89 \mathrm{~cm}$ & $0.9(0.4-2.2)$ & $0.7(0.4-1.3)$ & $1.6(0.9-2.6)$ \\
\hline Free Testosterone $>6.8 \mathrm{pg} / \mathrm{mL}$ & $4.2(0.5-33.2)$ & $2.2(1.0-4.9)$ & $1.8(0.8-4.0)$ \\
\hline Total Testosterone $>53$ ng/dL & $0.9(0.2-3.6)$ & $1.5(0.6-3.3)$ & $1.1(0.5-2.6)$ \\
\hline Abnormal DHEAS & $1.1(0.4-3.0)$ & $1.0(0.5-2.1)$ & $0.7(0.4-1.3)$ \\
\hline Acanthosis Nigricans Present & $0.7(0.3-1.5)$ & $1.0(0.6-1.6)$ & $1.4(0.9-2.3)$ \\
\hline
\end{tabular}

i. The largest available ovarian volume and single ovary follicle number was used

ii. Adjusted OR were controlled for age

iii. DHEAS measurements were performed at multiple laboratory sites, the reference cutoff for each site was used to determine normal/abnormal status

iv. HOMA-IR cutoff chosen to reflect more significant insulin resistance [22]

v. Fasting Insulin cutoff chosen to reflect the reference range of the laboratory used by majority of patients in the study

a indicates $p<0.05$

\section{Results}

\section{Study population}

Four-hundred thirty-nine patients seen in the PCOS clinic during the study time period consented for inclusion in the study. Of those, 355 were confirmed to have met Rotterdam criteria for PCOS. Fourtytwo subjects meeting Rotterdam criteria were excluded because they did not have complete ultrasound data. Three-hundred thirteen patients meeting Rotterdam criteria for PCOS and having sufficient ovarian measurements for analysis comprised the study population.

Of the included patients, $89 \%$ met the Rotterdam FN criteria for PCO and $52 \%$ met the OV criteria (Table 1). Thirty-four patients had a FN $<12(8.88 \pm 2.39), 279$ patients had a FN $\geq 12(22.42 \pm 9.69)$. One-hundred fiftyone patients had an OV of $\leq 10$ cc $(6.64 \pm 1.97), 162$ patients had an OV $>10$ cc $(14.11 \pm 5.73)$. Two-hundred twenty-seven patients had a FN $<25(16.11 \pm 4.5)$ and 86 had a FN $\geq 25(33.7 \pm 9.6)$. All groups were similar with respect to age, but patients with $\mathrm{OV}>10 \mathrm{cc}$ had higher mean BMI, waist circumference, fasting insulin and HOMA-IR (Tables 1 and 2).

Table 3 Logistic Regression Analysis of Follicle Number as a Continuous Variable

\begin{tabular}{lll}
\hline & AOR $(95 \% \mathrm{Cl})$ & $p$ \\
\hline Fasting Insulin $>19 \mu \mathrm{IU} / \mathrm{mL}$ & $1.00(0.97-1.03)$ & 0.81 \\
HOMA-IR $>4$ & $1.01(0.98-1.04)$ & 0.65 \\
Fasting Glucose $>100 \mathrm{mg} / \mathrm{dL}$ & $0.98(0.93-1.02)$ & 0.30 \\
2 h Glucose $>140 \mathrm{mg} / \mathrm{dL}$ & $0.97(0.92-1.01)$ & 0.16 \\
Waist Circ $>89 \mathrm{~cm}$ & $0.98(0.95-1.00)$ & 0.07 \\
\hline
\end{tabular}

i. Adjusted OR were controlled for age

\section{Follicle number}

The analysis of FN as a dichotomous variable, controlling for age, indicates that FN as defined by Rotterdam criteria does not predict abnormal metabolic indices among PCOS women in our study (Table 2). We performed an additional regression analysis examining FN as a continuous variable to confirm that the predetermined cut offs were not artificially concealing a difference. There was no significant relationship between FN as a continuous variable and any of the metabolic indices (Table 3).

\section{Ovarian volume}

The logistic regression analysis of OV as a dichotomous variable, controlling for age, indicates that the odds of an abnormal fasting insulin was 1.8 and the odds of an abnormal HOMA- IR 1.9 for patients with a maximum $\mathrm{OV}>10 \mathrm{cc}$ compared with patients with a maximum ovarian volume $\leq 10 \mathrm{cc}$. The relationship of OV with fasting glucose and 2-h glucose was not significant (Table 2).

Controlling for BMI attenuated the relationship between insulin resistance and OV such that it was no longer significant (data not shown). However, BMI and insulin resistance are highly co-linear. Further physiologic evidence suggests that insulin resistance may be on the causal pathway linking BMI and OV due to the known stimulatory effect of insulin on ovarian thecal cells [13]. For these reasons, a decision was made not to use BMI in the final model.

We also examined the relationship between ovarian features and abnormal waist circumference. Similar to the other markers of insulin resistance, OV was related to increased waist circumference (although this finding 
did not reach significance) while FN was not. The OR of having a waist circumference $>89 \mathrm{~cm}$ in patients with and $\mathrm{OV}>10$ cc was $1.6(0.9-2.6, p 0.08)$ compared to patients with an $\mathrm{OV} \leq 10 \mathrm{cc}$. For FN $\geq 12$ vs $<12$ the unadjusted OR was 0.9 (95\% CI $0.4-2.2, p 0.9$ ).

\section{PCO morphology and hyperandrogenism}

In our subgroup analysis of the 150 participants with banked serum ( $48 \%$ of all participants), we found no association of $\mathrm{OV}$ with elevated free or total testosterone. FN when treated as a dichotomous variable of $\geq 12$ vs $<12$ was also not associated with hyperandrogenemia. When FN $\geq 25$ vs $<25$ was examined, however, a FN $\geq 25$ was positively associated with free testosterone $>6.8 \mathrm{pg} /$ $\mathrm{mL}$ (Table 2).

\section{Discussion}

PCOS is a common endocrinopathy with a highly heterogeneous presentation. The association of PCOS with insulin resistance is well-known, however there is a lack of understanding regarding which patients with PCOS are most at risk of suffering adverse metabolic consequences. We investigated whether transvaginal ultrasound findings can be used to identify women with concurrent abnormal metabolic phenotype.

In our cohort, women with PCOS and OV $>10 \mathrm{cc}$ were 2 times more likely than those with $\mathrm{OV} \leq 10 \mathrm{cc}$ to exhibit biochemical markers of insulin resistance. Conversely, we found that although the vast majority of patients with PCOS meet the FN criteria ( $>12$ follicles per ovary), this finding is not associated with any abnormal metabolic parameters. Similarly, subjects meeting the newly recommended FN cutoff of 25 did not display more metabolic abnormalities than those not meeting this criteria. In summary, ovarian volume, but not follicle number, appears to predict metabolic abnormalities in a population of women with PCOS.

To date, there has been limited investigation regarding the individual components of PCO morphology and metabolic disease in adult women. One study examining the relationship of FN and OV with androgen levels and metabolic markers in women with PCOS $(n=88)$ failed to identify a correlation between $\mathrm{OV}$ or FN and indices of insulin resistance [28]. However, other smaller reports have suggested a relationship. In a study of 50 women with PCOS, Carmina showed that OV correlates with serum insulin $(r=.37, P<.01)$ and the Quicki $(r=-.31$. $P<.05)$ but does not correlate with BMI, LH, FSH or circulating androgens [29]. Among adolescents, Villa compared ovarian volume between 86 girls with PCOS and 48 controls, demonstrating that $\mathrm{OV}$ was associated with circulating insulin levels and markers of insulin resistance [30]. To our knowledge, our study is the largest study of women with PCOS to report the association between ovarian volume and markers of insulin resistance.

While there are multiple proposed explanations surrounding the pathophysiology of PCOS, it is well accepted that insulin resistance is a major driver of the metabolic phenotype and is compounded by obesity $[8$, 10]. Insulin resistance is likely also a key contributor to the PCOS reproductive phenotype. Systemic hyperinsulinemia due to peripheral insulin resistance provides a mitogenic stimulation of ovarian theca cells leading to an expansion of the theca cell compartment and increased androgen production [31-33]. Additionally, insulin has been shown to lower sex hormone binding globulin, which can lead to increased free androgen levels and clinical hyperandrogenism. In turn, biochemical hyperandrogenemia likely exacerbates peripheral insulin resistance [10]. The insulin-mediated theca cell expansion likely leads to increased stromal volume within the ovary. Our data reinforce the idea that increased OV may be serving as a measurable biomarker of increased levels of circulating insulin.

It is important to note that this cohort consisted exclusively of women with a confirmed diagnosis of PCOS. While some prior studies of ovulatory women meeting PCO morphology criteria have indicated an increased risk of higher fasting insulin and possibly insulin resistance [34], the majority of the data has not demonstrated an increased risk of metabolic dysfunction in regularly cycling women who meet only the follicle number or volume criteria for PCO [17, 34-37]. It is possible that the ovaries of women without PCOS who meet ovarian volume criteria are enlarged due to increased follicular mass rather than the increased theca cell/stromal mass possibly explaining the lack of association between ovarian volume and metabolic findings in a non-PCOS population. To date, there are no recommendations for ovulatory women meeting PCO criteria to undergo metabolic screening beyond the routine for their age and clinical risk factors.

Also important to consider is that cutoff for HOMA-IR in our study was 4 , which is higher than in some similar studies. After many population based studies, there is no single set value for HOMA-IR that is consistently used to define insulin resistance [22]. In determining which value we would use, we intentionally selected a value intended to identify more significant cases of insulin resistance.

Our study is strengthened by the systematic way in which all patients were examined and diagnosed under the supervision of one of two attending physicians. The clinic structure and data collection methodology remained unchanged over the time that subjects were recruited. Further, our ability to use banked serum to test all subjects' testosterone concentrations at a single laboratory using a single, well-validated RIA technique strengthens our findings related to hyperandrogenemia. 
Our study is potentially limited by a lack of heterogeneity in FN, skewed heavily towards numbers exceeding the Rotterdam cut off of 12. It is also important to note that Rotterdam FN criteria were used in diagnosing our patients with PCOS, which may be overly inclusive in light of current ultrasound technology and new guidelines recommending higher FN cutoffs. Finally, our sample size was insufficient to conclusively determine if $\mathrm{OV}$ has an effect on metabolic factors independent of BMI.

\section{Conclusions}

In summary, among the PCOS women in our study with an $\mathrm{OV}>10 \mathrm{cc}$ the odds ratio for abnormal biochemical markers of insulin resistance was two times that of women with normal $\mathrm{OV}$, indicating that $\mathrm{OV}$ is an important factor associated with metabolic risk in women with PCOS. In contrast, follicle number was not associated with clinical evidence of insulin resistance. Ovarian volume may thus serve as a physical biomarker of systemic hyperinsulinemia and the measurement of $\mathrm{OV}$ may provide a useful tool to aid clinicians in their risk stratification and counseling of patients with PCOS.

\section{Abbreviations \\ BMI: Body mass index; CHR: Committee on Human Research; FN: Follicle number; FPG: Fasting plasma glucose; FPI: Fasting plasma insulin; HOMA- IR: Homeostasis model assessment-estimated insulin resistance: LH: Luteinizing hormone; mFG: Modified Ferriman-Gallwey; NHANES: National Health and Nutrition Examination Survey; OR: Odds ratio; OV: Ovarian volume; PCOM: Polycystic ovary morphology; PCOS: Polycystic ovary syndrome; RIA: Radioimmunoassay; SHBG: Sex hormone binding globulin; UCSF: University of California, San Francisco; UVA: University of Virginia}

\section{Acknowledgments}

The authors thank Dr Lee Zane, Lili Kuzmich and Gina Davis for their contributions to the care of our patients and the collection of data for this study.

\section{Funding}

The University of Virginia Center for Research in Reproduction Ligand Assay and Analysis Core is supported by the Eunice Kennedy Shriver NICHD/NIH (SCCPIR) Grant U54-HD28934.

\section{Availability of data and materials}

The dataset supporting the conclusions of this article is not publically available.

\section{Authors' contributions}

SRP, MIC, CNK and HGH designed the study. SRP, LP, KS, MIC and HGH examined patients in the PCOS clinic and collected and entered data. CNK provided statistical support. SRP drafted the manuscript. All authors revised and corrected the drafts and approved the final version of the manuscript.

\section{Competing interests}

None of the above authors have any disclosures, financial or otherwise, to make. An abstract involving a portion of the data from this submission was presented at the 2012 meeting of the American Society of Reproductive Medicine.

\section{Consent for publication}

Not applicable

\section{Ethics approval and consent to participate}

The Committee on Human Research (CHR), which serves as the Institutional Review Board for the University of California at San Francisco, must approve all research involving human subjects at the UCSF. The CHR approved the research protocol and all data collection instruments employed in this study.

\section{Publisher's Note}

Springer Nature remains neutral with regard to jurisdictional claims in published maps and institutional affiliations.

\section{Author details}

${ }^{1}$ Center for Reproductive Health, University of California at San Francisco, 2356 Sutter Street, San Francisco 94115, CA, USA. ${ }^{2}$ Dermatology, University of California at San Francisco, 1701 Divisadero, San Francisco 94115, CA, USA.

Received: 8 November 2016 Accepted: 13 April 2017

Published online: 30 May 2017

\section{References}

1. Knochenhauser ES, Key TJ, Kahsar-Miller M, Waggoner W, Boots LR, Azziz R. Prevalence of the polycystic ovary syndrome in unselected black and white women of southeastern United States: a prospective study. J Clin Endocrinol Metab. 1998;83:3078-82.

2. Franks S. Polycystic ovary syndrome. N Engl J Med. 1995;333:853-61.

3. Azziz R, Woods KS, Reyna R, Key TJ, Knochenhauer ES, Yildiz BO. The prevalence and features of the polycystic ovary syndrome in an unselected population. J Clin Endocrinol Metab. 2004;89:2745-9.

4. March WA, Moore VM, Willson KJ, Phillips DI, Norman RJ, Davies MJ. The prevalence of polycystic ovary syndrome in a community sample assessed under contrasting diagnostic criteria. Hum Reprod. 2010;25(2):544-51.

5. Eilertsen TB, Vanky E, Carlsen SM. Anti-Mullerian hormone in the diagnosis of polycystic ovary syndrome: can morphologic description be replaced? Hum Reprod. 2012;27:2494-502.

6. Lauritsen MP, Bentzen JG, Pinborg A, Loft A, Forman JL, Thuesen LL, et al. The prevalence of polycystic ovary syndrome in a normal population according to the Rotterdam criteria versus revised criteria including antiMullerian hormone. Hum Reprod. 2014;29:791-801.

7. Rotterdam ESHRE/ASRM-Sponsored PCOS Consensus Workshop Group. Revised 2003 consensus on diagnostic criteria and long-term health risks related to polycystic ovary syndrome. Fertil Steril. 2004;81:19-25.

8. Burghan GA, Givens JR, Kitabchi AE. Correlation of hyperandrogenism with hyperinsulinism in polycystic ovarian disease. J Clin Endocrinol Metab. 1980; 50:113-6.

9. Dunaif A, Segal KR, Futterweit W, Dobrjansky A. Profound peripheral insulin resistance, independent of obesity, in polycystic ovary syndrome. Diabetes. 1989:38:1165-74.

10. Diamanti-Kandarakis E, Dunaif A. Insulin resistance and the polycystic ovary syndrome revisited: an update on mechanisms and implications. Endocr Rev. 2012;33:981-1030

11. Marcondes JA, Hayashida SA, Barcellos CR, Rocha MP, Maciel GA, Baracat EC. Metabolic syndrome in women with polycystic ovary syndrome: prevalence, characteristics and predictors. Arq Bras Endocrinol Metabol. 2007;51:972-9.

12. Kim J, Chae YK, Chernoff A. The risk for coronary heart disease according to insulin resistance with and without type 2 diabetes. Endocr Res. 2013;38: 195-205.

13. Palaniappan M, Menon B, Menon KM. Stimulatory effect of insulin on thecainterstitial cell proliferation and cell cycle regulatory proteins through MTORC1 dependent pathway. Mol Cell Endocrinol. 2013;366(1):81-9.

14. Franks $\mathrm{S}$, Gilling-Smith $\mathrm{C}$, Watson $\mathrm{H}$, Willis $\mathrm{D}$. Insulin action in the normal and polycystic ovary. Endocrinol Metab Clin North Am. 1999;28:361-78.

15. Dewailly D, Lujan ME, Cedars MI, Laven J, Norman RJ, Escobar-Morreale HF. Definition and significance of polycystic ovarian morphology: a task force report from the Androgen Excess and Polycystic Ovary Syndrome Society. Hum Reprod Update. 2014;20:334-52.

16. Dewailly D, Pigny P, Soudan B, Catteau-Jonard S, Decanter C, Poncelet E, et al. Reconciling the definitions of polycystic ovary syndrome: the ovarian follicle number and serum anti-Mullerian hormone concentrations aggregate with the markers of hyperandrogenism. J Clin Endocrinol Metab. 2010;95:4399-405.

17. Villarroel C, Merino PM, Lopez P, Eyzaguirre FC, Van Velzen A, Iniquez G, et al. Polycystic ovarian morphology in adolescents with regular menstrual cycles is associated with elevated anti-Mullerian hormone. Hum Reprod. 2011;26:2861-8 
18. Johnstone EB, Rosen MP, Neril R, Trevithick D, Sternfeld B, Murphy R, et al. The polycystic ovary post-rotterdam: a common, age-dependent finding in ovulatory women without metabolic significance. J Clin Endocrinol Metab. 2010;95:4965-72.

19. Najmabadi S, Wilcox JG, Acacio BD, Thornton MH, Kolb BA, Paulson RJ. The significance of polycystic-appearing ovaries versus normal-appearing ovaries in patients with polycystic ovary syndrome. Fertil Steril. 1997;67:631-5.

20. Guastella E, Longo RA, Carmina E. Clinical and endocrine characteristics of the main polycystic ovary syndrome phenotypes. Fertil Steril. 2010;94:2197-201.

21. Moran $L$, Teede H. Metabolic features of the reproductive phenotypes of polycystic ovary syndrome. Hum Reprod Update. 2009;15:477-88.

22. Stern SE, Williams K, Ferrannini E, Defronzo RA, Bogardus C, Stern MP. Identification of individuals with insulin resistance using routine clinical measurements. Diabetes. 2005;54(2):333-9.

23. Quinn M, Shinkai K, Pasch L, Kuzmich L, Cedars M, Huddleston H. Prevalence of androgenic alopecia in patients with polycystic ovary syndrome and characterization of associated clinical and biochemical features. Fertil Steril. 2014;101:1129-34

24. Azziz R, Carmina E, Dewailly D, Diamanti-Kandarakis E, Escobar- Morreale HF, Futterweit W, et al. The Androgen Excess and PCOS Society criteria for the polycystic ovary syndrome: the complete task force report. Fertil Steril. 2009; 91:456-88.

25. Matthews DR, Hosker JP, Rudenski AS, Naylor BA, Treacher DF, Turner RC. Homeostasis model assessment: insulin resistance and beta-cell function from fasting plasma glucose and insulin concentrations in man. Diabetologia. 1985;28:412-9.

26. Rinaldi S, Geay A, Dechaud H, Biessy C, Zeleniuch-Jacquotte A, Akhmedkhanov A, et al. Validity of free testosterone and free estradiol determinations in serum samples from postmenopausal women by theoretical calculations. Cancer Epidemiol Biomarkers Prev. 2002;11:1065-71.

27. Vermeulen A, Verdonck L, Kaufman JM. A critical evaluation of simple methods for the estimation of free testosterone. J Clin Endocrinol Metab. 1999;84:3666-72.

28. Legro RS, Chiu P, Kunselman AR, Bentley CM, Dodson WC, Dunaif A. Polycystic ovaries are common in women with hyperandrogenic chronic anovulation but do not predict metabolic or reproductive phenotype. J Clin Endocrinol Metab. 2005;90:2571-9.

29. Carmina E, Orio F, Palomba S, Longo RA, Lombardi G, Lobo RA. Ovarian size and blood flow in women with polycystic ovary syndrome and their correlations with endocrine parameters. Fertil Steril. 2005;84:413-9.

30. Villa P, Rossodivita A, Sagnella F, Moruzzi MC, Mariano N, Lassandro AP, et al. Ovarian volume and gluco-insulinaemic markers in the diagnosis of PCOS during adolescence. Clin Endocrinol. 2013;78:285-90.

31. Duleba AJ, Spaczynski RZ, Olive DL. Insulin and insulin-like growth factor I stimulate the proliferation of human ovarian theca-interstitial cells. Fertil Steril. 1998;69:335-40.

32. Will MA, Palaniappan M, Peefel H, Kayampilly P, Menon KM. Metformin: direct inhibition of rat ovarian theca-interstitial cell proliferation. Fertil Steril. 2012;98:201-14.

33. Tosi F, Negri C, Perrone F, Dorizzi R, Castello R, Bonora E, et al. Hyperinsulinemia amplifies GnRH agonist stimulated ovarian steroid secretion in women with polycystic ovary syndrome. J Clin Endocrinol Metab. 2012:97:1712-9.

34. Adams JM, Taylor AE, Crowley Jr WF, Hall JE. Polycystic ovarian morphology with regular ovulatory cycles: insights into the pathophysiology of polycystic ovarian syndrome. J Clin Endocrinol. 2004;89:4343-50.

35. Michelmore KF, Balen AH, Dunger DB, Vessey MP. Polycystic ovaries and associated clinical and biochemical features in young women. Clin Endocrinol. 1999;51:779-86.

36. Carmina E, Wong L, Chang L, Paulson RJ, Sauer MV, Stanczyk FZ, et al. Endocrine abnormalities in ovulatory women with polycystic ovaries on ultrasound. Hum Reprod. 1997;12:905-9.

37. Chang PL, Lindheim SR, Lowre C, Ferin M, Gonzalez F, Berglund L. Normal ovulatory women with polycystic ovaries have hyperandrogenic pituitaryovarian responses to gonadotropin-releasing hormone-agonist testing. J Clin Endocrinol Metab. 2000;85:995-1000.

\section{Submit your next manuscript to BioMed Central and we will help you at every step:}

- We accept pre-submission inquiries

- Our selector tool helps you to find the most relevant journal

- We provide round the clock customer support

- Convenient online submission

- Thorough peer review

- Inclusion in PubMed and all major indexing services

- Maximum visibility for your research

Submit your manuscript at www.biomedcentral.com/submit
Biomed Central 